\title{
Curvature-Restored Gauge Invariance and Ultraviolet Naturalness
}

\author{
Durmuş Ali Demir \\ Department of Physics, Izmir Institute of Technology (IZTECH), 35430 İmir, Turkey \\ Correspondence should be addressed to Durmuş Ali Demir; demir@physics.iztech.edu.tr
}

Received 20 July 2016; Accepted 15 November 2016

Academic Editor: Elias C. Vagenas

Copyright (C) 2016 Durmus Ali Demir. This is an open access article distributed under the Creative Commons Attribution License, which permits unrestricted use, distribution, and reproduction in any medium, provided the original work is properly cited. The publication of this article was funded by SCOAP

It is shown that $\left(a \Lambda^{2}+b|H|^{2}\right) R$ in a spacetime of curvature $R$ is a natural ultraviolet (UV) completion of $\left(a \Lambda^{4}+b \Lambda^{2}|H|^{2}\right)$ in the flat-spacetime Standard Model (SM) with Higgs field $H$, UV scale $\Lambda$, and loop factors $a$ and $b$. This curvature completion rests on the fact that $\Lambda$-mass gauge theory in flat spacetime turns, on the cut view $R=4 \Lambda^{2}$, into a massless gauge theory in curved spacetime. It provides a symmetry reason for curved spacetime, wherein gravity and matter are both low-energy effective phenomena. Gravity arises correctly if new physics exists with at least 63 more bosons than fermions, with no need to interact with the SM and with dark matter as a natural harbinger. It can source various cosmological, astrophysical, and collider phenomena depending on its spectrum and couplings to the SM.

SM, spectrally completed with the discovery of its Higgs boson, is experimentally affirmed to describe physics at the Fermi scale, $G_{F}$. Its validity in the UV direction comes to an end at a physical scale $\Lambda$, at which it is environed by new UV physics. Integrating out all trans-Fermi high-frequency fluctuations, low-energy SM fields $\psi_{\mathrm{SM}}$ develop the effective action

$$
S(\eta)=S_{G_{F}}\left(\eta, \psi_{\mathrm{SM}}, \log \left(G_{F} \Lambda^{2}\right)\right)+S_{\Lambda}^{0}(\eta)+S_{\Lambda}^{1}(\eta)
$$

in the flat spacetime of metric $\eta_{\mu \nu}$ such that the action $S_{G_{F}}(\eta$, $\left.\psi_{\mathrm{SM}}, \log \left(G_{F} \Lambda^{2}\right)\right)$ encodes the tree-level SM interactions augmented by logarithmic UV contributions.

$$
S_{\Lambda}^{0}(\eta)=\int d^{4} x \sqrt{\|\eta\|}\left\{a \Lambda^{4}+a_{m} \Lambda^{2} m_{H}^{2}+b \Lambda^{2} H^{\dagger} H\right\}
$$

entrains a UV-sized vacuum energy along with a UV-sized Higgs boson mass, and

$$
S_{\Lambda}^{1}(\eta)=\int d^{4} x \sqrt{\|\eta\|} c_{V} \Lambda^{2} \eta_{\mu \nu} \operatorname{Tr}\left\{V^{\mu} V^{\nu}\right\}
$$

adds UV-sized gauge boson masses so that hypercharge, isospin, and color are all explicitly broken at the UV [1].

In the above, $\Lambda$ is a fundamental scale of nature just as $G_{F}$. It is a physical scale rather than a formal momentum cutoff introduced to regulate the loop integrals. In this sense, $S(\eta)$ is a physical effective theory whose no part, including $S_{\Lambda}^{0}(\eta)$ and $S_{\Lambda}^{1}(\eta)$, can be modified at will. If $\Lambda$ were a formal cutoff it would be possible to eradicate both $S_{\Lambda}^{0}(\eta)$ and $S_{\Lambda}^{1}(\eta)$ simply by switching from cutoff regularization to, say, dimensional regularization.

Quarks and leptons, whose masses vary with $\Lambda$ only logarithmically, stay put at the Fermi scale. In view of the Higgs mechanism, therefore, $S_{\Lambda}^{0}(\eta)$ and $S_{\Lambda}^{1}(\eta)$ stand as subversive outliers. They render the SM unnatural [2-5]. Its natural extensions like supersymmetry, extra dimensions, and technicolor have not even glimpsed at collider searches [6]. It must therefore be naturalized by a different mechanism. That mechanism, if exists, must be able to (i) eradicate $S_{\Lambda}^{1}(\eta)$ to restore gauge invariance, (ii) ameliorate $S_{\Lambda}^{0}(\eta)$ to stabilize the Higgs sector, and (iii) elucidate $\Lambda$ to reveal the UV physics behind it. They are approached below by restoring gauge invariance (not by using affine geometry as in [7], though the results agree). 
In search for a mechanism to restore gauge symmetries at the UV, it proves effectual to introduce

$$
I(\eta)=\int d^{4} x \sqrt{\|\eta\|} c_{V} \operatorname{Tr}\left\{\eta_{\mu \nu} \eta_{\alpha \beta} V^{\mu \alpha} V^{\nu \beta}\right\}
$$

and its by-parts arrangement for $R_{\xi}$ gauge

$$
\begin{aligned}
& \widetilde{I}(\eta) \\
& =\int d^{4} x \sqrt{\|\eta\|} 2 c_{V} \operatorname{Tr}\left\{V^{\mu}\left(-\mathscr{D}^{2} \eta_{\mu \nu}+\mathscr{D}_{\mu} \mathscr{D}_{\nu}+V_{\mu \nu}\right) V^{\nu}\right\}
\end{aligned}
$$

as two gauge kinetic functionals which convert to one another through by-parts integration. This by-parts equivalence of theirs, $\widetilde{I}(\eta) \equiv I(\eta)$, can be put in use to construct

$$
\widetilde{S}_{\Lambda}^{1}(\eta)=-\frac{1}{2} I(\eta)+\frac{1}{2} \widetilde{I}(\eta)+S_{\Lambda}^{1}(\eta)
$$

as an equivalent of $S_{\Lambda}^{1}(\eta)$. Corresponding effective action

$$
\widetilde{S}(\eta)=S_{G_{F}}\left(\eta, \psi_{S M}, \log \left(G_{F} \Lambda^{2}\right)\right)+S_{\Lambda}^{0}(\eta)+\widetilde{S}_{\Lambda}^{1}(\eta)
$$

is equivalent to $S(\eta)$. This dynamical equivalence is, however, quite vulnerable to spacetime geometry, indeed viewing $\eta_{\mu \nu}$ as a specific value assigned to a curved metric $g_{\mu \nu}$ and inductively unassigning it as

$$
\eta_{\mu \nu} \preccurlyeq g_{\mu \nu}
$$

$\widetilde{S}_{\Lambda}^{1}(\eta)$, as is, can be carried into curved spacetime to find that $\widetilde{S}_{\Lambda}^{1}(g) \not \equiv S_{\Lambda}^{1}(g)$ simply because $\widetilde{I}(g) \not \equiv I(g)$. The reason is that $\widetilde{I}(g)$, unlike $\widetilde{I}(\eta)$, must involve not just $V_{\mu \nu}$ but also $V_{\mu \nu}+R_{\mu \nu}(g)$, where $R_{\mu \nu}(g)$ is the Ricci curvature of $g_{\mu \nu}$. This nonequivalence is actually a blessing in disguise for taming the gauge sector. Indeed, if $\Lambda^{2} g_{\mu \nu}$ in $S_{\Lambda}^{1}(g)$ is construed as a specific value assigned to $R_{\mu \nu}(g)$, then its inductive unassignment

$$
\Lambda^{2} g_{\mu \nu} \preccurlyeq R_{\mu \nu}(g)
$$

takes $S_{\Lambda}^{1}(g)$ into

$$
S_{\Lambda}^{1}(R)=\int d^{4} x \sqrt{\|g\|} c_{V} R_{\mu \nu}(g) \operatorname{Tr}\left\{V^{\mu} V^{\nu}\right\}
$$

with which $S_{\Lambda}^{1}(R)+(1 / 2) \widetilde{I}(g)=(1 / 2) I(g)$ and thus

$$
\widetilde{S}_{\Lambda}^{1}(R)=-\frac{1}{2} I(g)+\frac{1}{2} \widetilde{I}(g)+S_{\Lambda}^{1}(R) \equiv 0
$$

so that all gauge symmetries are restored at the UV. The unassignment of $g_{\mu \nu}\left(R_{\mu \nu}\right)$ renders the Poincare (gauge) breaking by $\Lambda$ futile. The classical curvature restores gauge invariance to naturalize the SM gauge sector.

This naturalization mechanism extends over entirety of the SM if $\widetilde{S}(\eta)$ is construed as a curved spacetime effective action $\widetilde{S}(R)$ evaluated at the curvature $R_{\mu \nu}(g)=\Lambda^{2} g_{\mu \nu}$ and then at the metric $g_{\mu \nu}=\eta_{\mu \nu}$. Namely, $\widetilde{S}(\eta)$ is nothing but the UV scale cut view of $\widetilde{S}(R)$ :

$$
\widetilde{S}(\eta)=\widetilde{S}(R) \mid \begin{gathered}
\text { set first } \\
R_{\mu \nu}(g)=\Lambda^{2} g_{\mu \nu} \\
\text { and then } \\
g_{\mu \nu}=\eta_{\mu \nu}
\end{gathered}
$$

such that $\widetilde{S}(\eta)$ and $\widetilde{S}(R)$ both embrace $\Lambda$, albeit with different physical meanings. Physically, $\widetilde{S}(R)$ must involve

(a) no extra couplings not found in $\widetilde{S}(\eta)$ as no quantum fluctuations are left to induce any new coupling,

(b) no extra forces except gravity as spacetime can attain required elasticity if $\Lambda$ nears gravitational scale [8],

so that it comes to take the familiar Einstein-Hilbert form

$$
\begin{aligned}
\widetilde{S}(R)= & S_{G_{F}}\left(g, \psi_{\mathrm{SM}}, \log \left(G_{F} \Lambda^{2}\right)\right) \\
& +\int d^{4} x \sqrt{\|g\|}\left(a \Lambda^{2}+a_{m} m_{H}^{2}+b H^{\dagger} H\right) \frac{R(g)}{4}
\end{aligned}
$$

though already at one loop (see [7] for a variant study).

$$
a=\frac{1}{64 \pi^{2}}\left(n_{b}-n_{f}\right)
$$

is negative for $n_{b}=28$ bosonic and $n_{f}=90$ fermionic degrees of freedom in the SM. This means that gravity can be induced properly only if the SM is extended by

$$
n_{b}^{\mathrm{NP}}-n_{f}^{\mathrm{NP}} \geq 63
$$

new fields belonging to some "new physics" (NP) sector lying at a scale $G_{\mathrm{NP}}$. Its effective action is of the form

$$
\begin{aligned}
\widetilde{S}^{\mathrm{NP}}(\eta)= & S_{\mathrm{G}_{\mathrm{NP}}}\left(\eta, \psi_{\mathrm{NP}}, \log \left(G_{\mathrm{NP}} \Lambda^{2}\right)\right)+S_{\Lambda}^{0, \mathrm{NP}}(\eta) \\
& +\widetilde{S}_{\Lambda}^{1, \mathrm{NP}}(\eta)
\end{aligned}
$$

if it is secluded from the SM. If it has no scalars [2-5],

$$
S_{\Lambda}^{0, \mathrm{NP}}(\eta)=\int d^{4} x \sqrt{\|\eta\|} a^{\mathrm{NP}} \Lambda^{4},
$$

so that, through (12), $\widetilde{S}(\eta)$ and $\widetilde{S}^{\mathrm{NP}}(\eta)$ add up to give

$$
\begin{aligned}
\widetilde{S}_{\mathrm{SM}+\mathrm{NP}}(R) & \\
= & S_{G_{F}}\left(g, \psi_{\mathrm{SM}}, \log \left(G_{F} \Lambda^{2}\right)\right) \\
& +S_{G_{\mathrm{NP}}}\left(g, \psi_{\mathrm{NP}}, \log \left(G_{\mathrm{NP}} \Lambda^{2}\right)\right) \\
& +\int d^{4} x \sqrt{\|g\|}\left(\frac{R(g)}{16 \pi G_{N}}+\zeta_{H} R(g) H^{\dagger} H\right)
\end{aligned}
$$

as a completely UV-natural effective field theory governing $\psi_{\mathrm{SM}}$ and $\psi_{\mathrm{NP}}$ dynamics in curved spacetime [7] such that $\zeta_{H}=b / 4$ is Higgs-curvature direct coupling, and

$$
G_{N}=\left(4 \pi\left(a+a^{\mathrm{NP}}\right) \Lambda^{2}+4 \pi a_{m} m_{H}^{2}\right)^{-1}
$$


is Newton's constant (see also $[9,10])$. Not only these curvature couplings but also those in $S_{G_{F}}\left(g, \psi_{\mathrm{SM}}, \log \left(G_{F} \Lambda^{2}\right)\right)$ and $S_{G_{\mathrm{NP}}}\left(g, \psi_{\mathrm{NP}}, \log \left(G_{\mathrm{NP}} \Lambda^{2}\right)\right)$ are all given by couplings in the flat spacetime effective actions $\widetilde{S}(\eta)$ and $\widetilde{S}^{\mathrm{NP}}(\eta)$.

In (18), vacuum energy, already naturalized in the UV with corrections $\propto\left(m_{H}^{2}\right)^{2} \log \left(G_{F} \Lambda^{2}\right)$, needs to be naturalized also in the IR as it is still far bigger than the observational value of $m_{v}^{4}$. This is the IR cosmological constant problem $[9,10]$.

In (18), the NP, secluded from the SM, is a natural home to noninteracting dark matter. Its spectrum, modelable as $\mathrm{SU}(6)$ gauge theory or $\mathrm{SU}(7)$ with two fermions or anything with $n_{b}^{\mathrm{NP}}-n_{f}^{\mathrm{NP}} \geq 63$, is secluded enough to form a dark matter observable via only its weight [11].

In (18), there exist no higher-curvature terms because, after inducing the Einstein-Hilbert term, no suitable couplings are left in the SM. If needed, they can be added by hand but that reduces predictive power of SM + NP. Indeed, adding, for instance, the ghost-free quadratic action $\int d^{4} x \sqrt{\|g\|}\left\{\omega W^{2}(g)+\gamma\left(R(g)^{2}-6 G^{2}(g)\right)\right\}$ does not unnaturalize (18), yet this ambiguates it by the undetermined coefficients of Weyl $(\omega)$ and Gauss-Bonnet $(\gamma)$ invariants.

In (18), logarithmic UV contributions coming through $\log \left(G_{F} \Lambda^{2}\right)$ lead to multiplicative renormalizations of $S_{G_{F}}(g$, $\left.\psi_{\mathrm{SM}}, \log \left(G_{F} \Lambda^{2}\right)\right)$ and $S_{G_{\mathrm{NP}}}\left(g, \psi_{\mathrm{NP}}, \log \left(G_{\mathrm{NP}} \Lambda^{2}\right)\right)$. These logarithms can actually be construed as loop integrals in a $D=$ $4-\epsilon$-dimensional momentum space of total volume $\mu^{2 \epsilon} \infty^{4-2 \epsilon}$ so that the formal equivalence $\log \left(G_{F} \Lambda^{2}\right) \equiv 2 / \epsilon+\log G_{F} \mu^{2}$ with small but finite $\epsilon$ enables the two logarithmic parts of (18) to be formulated in the dimensional regularization scheme with associated renormalization methods [1].

In (18), SM is decoupled from NP. Their coupling can cause new effects. One possibility is Higgs mass shifts like $G_{\mathrm{NP}}^{-1 / 2} \log \left(G_{\mathrm{NP}} \Lambda^{2}\right)$, which beget logarithmic unnaturalness as in supersymmetry unless $G_{\mathrm{NP}} \cong G_{F}$ [12]. The interacting dark matter under search in direct detection experiments is another possibility. The recent LHC diphoton signal [13] can well be a precursor of such NP.

In (18), matter and gravity meet in a physically consistent framework in that they both are sub-Fermi effective interactions. This is a crucial property because putting quantized matter into curved geometry is an aporia as quantum gravity is distant and classical gravity is inconsistent $[14,15]$. They do not enhance naturalness $[16,17]$.

In summary, restoration of gauge invariance has led to naturalization of the SM via gravity. This is not surprising because, at least in macroscopic world, it is gravity that dictates what is natural and what is unnatural. It is in this sense that unnatural $\widetilde{S}(\eta)+\widetilde{S}^{\mathrm{NP}}(\eta)$ in flat spacetime turns into natural $\widetilde{S}_{\mathrm{SM}+\mathrm{NP}}(R)$ in curved spacetime. The mechanism makes, as were also with the previous work [7], three salient predictions:

(I) Gravity arises as a large-distance effective force consistently coupled to the low-energy quantum effective action. It is the requisite UV physics that completes the $\mathrm{SM}$ and renders the $\Lambda$ physical.
(II) New physics exists as a highly crowded sector $(\Lambda<$ $\left(8 \pi G_{N}\right)^{-1 / 2}$ for $\left.n_{b}^{\mathrm{NP}}-n_{f}^{\mathrm{NP}}>128 \pi^{2}+62 \approx 1325\right)$, which does not have to couple to the SM matter. This secluded sector, which can in principle lie at any scale sufficiently below $\Lambda$, can source dark matter as a noninteracting nonbaryonic matter which can be sensed via only its weight. In this setup, $\mathrm{SM}+\mathrm{NP}$ is all UV-natural.

(III) New physics may interact with the SM partly or wholly. In this case, scalar fields in the NP, even its vector-like fermions, can cause the Higgs boson mass to shift by $G_{\mathrm{NP}}^{-1 / 2}$ depending on coupling strengths. They destabilize the SM unless $G_{\mathrm{NP}} \cong G_{F}$. The LHC diphoton signal, if real, may be stemming from such NP sector.

\section{Additional Points}

Future research will reveal more about the formalism.

\section{Competing Interests}

The author declares that there are no competing interests regarding the publication of this paper.

\section{Acknowledgments}

This work is supported in part by the TÜBİTAK Grant 115F212. It is dedicated to honorable memory of Professor Namık K. Pak who was a dedicated teacher, an indefatigable researcher, and a great friend.

\section{References}

[1] M. E. Peskin and D. V. Schroeder, An Introduction to Quantum Field Theory, Addison-Wesley, Reading, Mass, USA, 1995.

[2] V. F. Weisskopf, "On the self-energy and the electromagnetic field of the electron," Physical Review, vol. 56, no. 1, pp. 72-85, 1939.

[3] L. Susskind, "Dynamics of spontaneous symmetry breaking in the Weinberg-Salam theory," Physical Review D, vol. 20, no. 10, p. 2619, 1979.

[4] M. J. G. Veltman, “The infrared-ultraviolet connection," Acta Physica Polonica B, vol. 12, no. 5, pp. 437-457, 1981.

[5] M. J. G. Veltman, "Reflections on the Higgs system," Cern Yellow Report, vol. 97, no. 5, pp. 1-25, 1997.

[6] LHC Exotica and SUSY results, https://twiki.cern.ch/.

[7] D. A. Demir, "A mechanism of ultraviolet naturalness," https:// arxiv.org/abs/1510.05570.

[8] A. D. Sakharov, "Vacuum quantum fluctuations in curved space and the theory of gravitation," Doklady Akademii Nauk Seri Fizik, vol. 177, pp. 70-71, 1967, reprinted in Soviet Physics Uspekhi vol. 34, p. 394, 1991.

[9] D. A. Demir, "Vacuum energy as the origin of the gravitational constant," Foundations of Physics, vol. 39, article 1407, 2009.

[10] D. A. Demir, "Stress-energy connection and cosmological constant problem," Physics Letters B, vol. 701, no. 4, pp. 496-502, 2011. 
[11] P. J. E. Peebles and A. Vilenkin, "Noninteracting dark matter," Physical Review D, vol. 60, no. 10, Article ID 103506, 1999.

[12] S. P. Maretin, "A supersymmetry primer," Advanced Series on Directions in High Energy Physics, vol. 18, pp. 1-98, 2010.

[13] ATLAS Collaboration, ATLAS-CONF-2016-018; CMS Coll., CMS-PAS-EXO-16-018.

[14] T. W. B. Kibble and S. Randjbar-Daemi, "Non-linear coupling of quantum theory and classical gravity," Journal of Physics A: Mathematical and Theoretical, vol. 13, no. 1, article 141, 1980.

[15] C. Carlip, "Is quantum gravity necessary?” Classical and Quantum Gravity, vol. 25, no. 15, Article ID 154010, 2008.

[16] G. F. Giudice, "Naturalness after LHC8," https://arxiv.org/abs/ 1307.7879.

[17] J. D. Wells, "Higgs naturalness and the scalar boson proliferation instability problem," Synthese, 2014. 

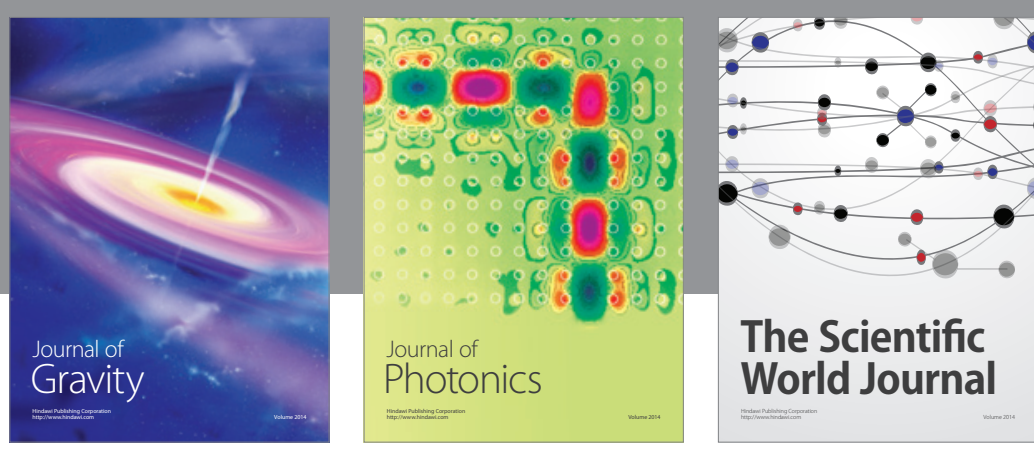

The Scientific World Journal
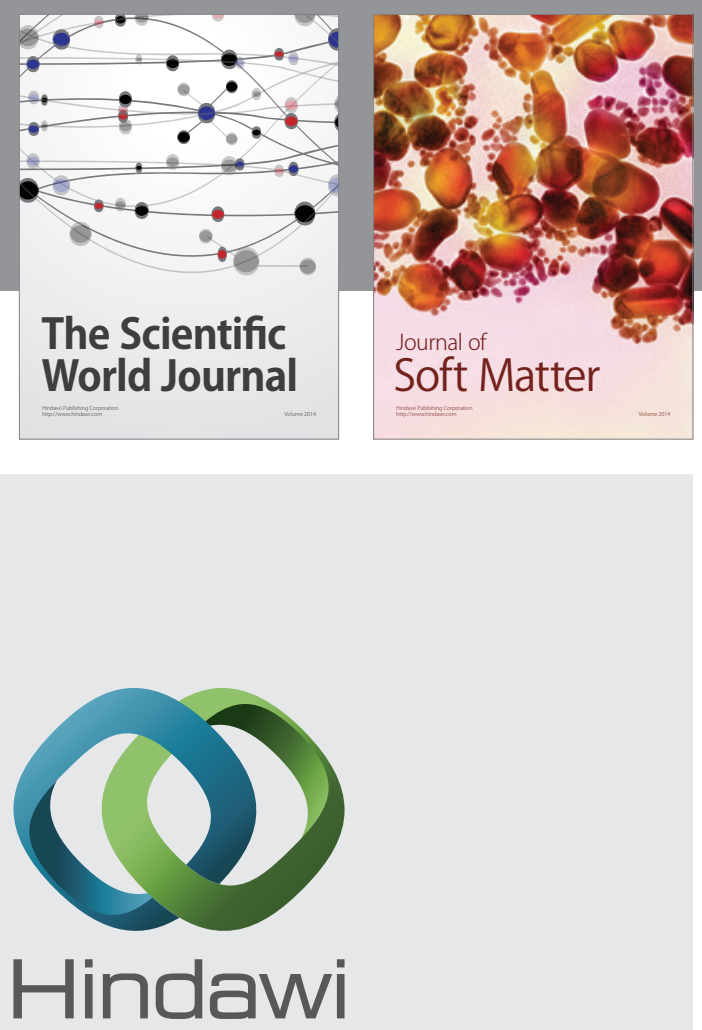

Submit your manuscripts at

http://www.hindawi.com

nternational Journal of

Statistical Mechanics
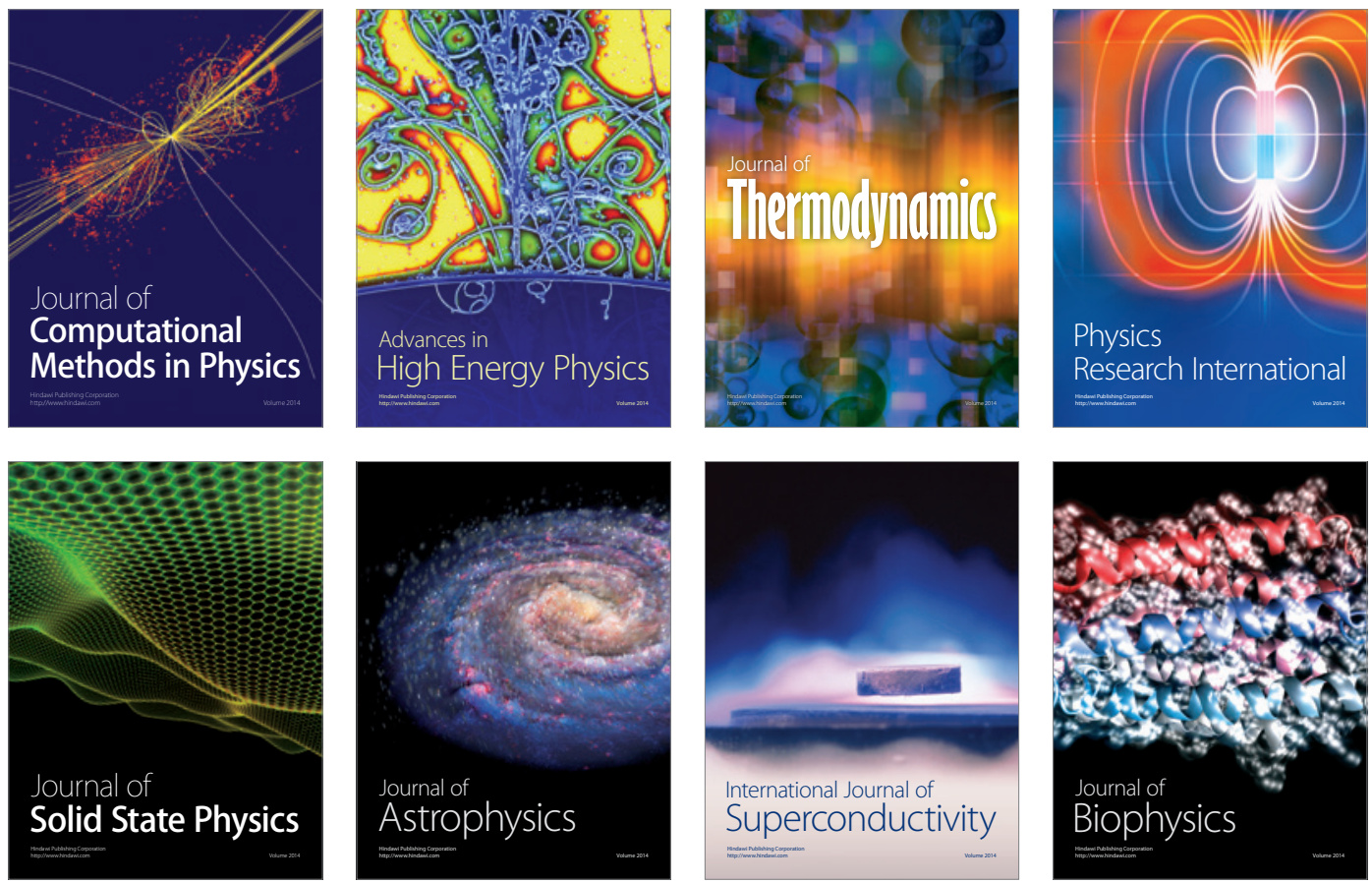
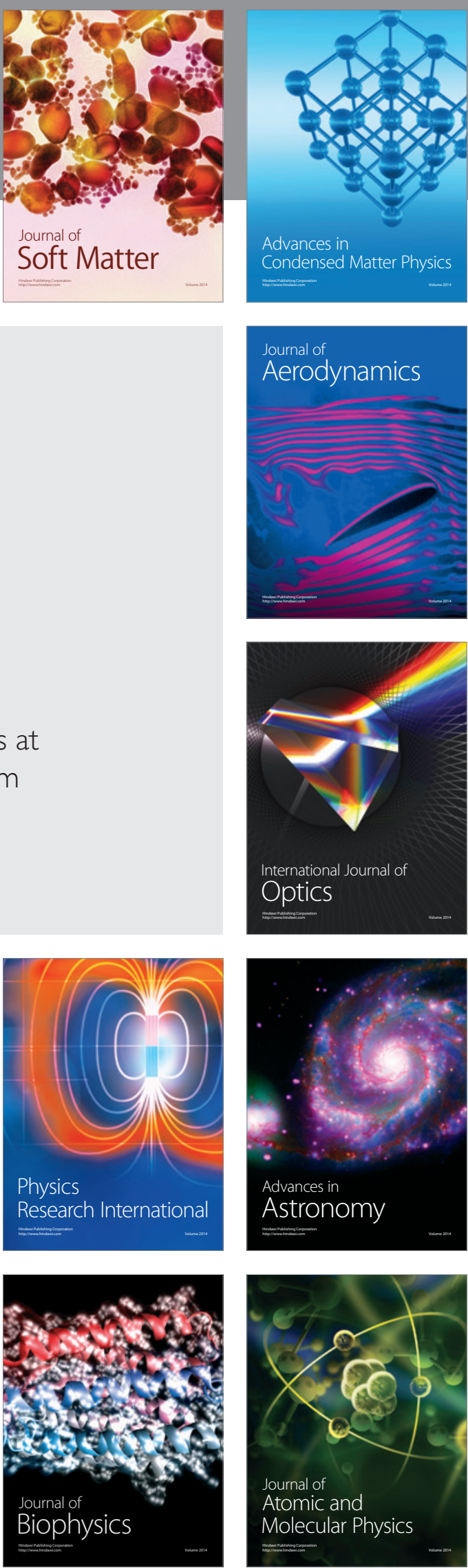